\title{
Relación entre ratio digital (2D:4D) y severidad preoperatoria del cáncer de próstata
}

\section{Relationship between Digit Ratio (2D:4D) and Preoperative Severity of Prostate Cancer}

\author{
Carlos Sánchez-Rodríguez ${ }^{1}$ Julián Oñate-Celdrán ${ }^{2}$ Damián García-Escudero ${ }^{1}$ Paula Samper-Mateo ${ }^{2}$ \\ Ana Vilchez-Costas ${ }^{2}$ Marcos Torres-Roca ${ }^{2}$ Ana Belén Maldonado-Cárceles ${ }^{3,4}$ José Juan López-Espín ${ }^{5,6}$ \\ Francisco Martínez-Díaz ${ }^{7}$ Jaime Mendiola-Olivares ${ }^{3,6}$ Alberto Manuel Torres-Cantero 3,4,6
}

\footnotetext{
${ }^{1}$ Departamento de Urología, Hospital Universitario los Arcos del Mar Menor, San Javier (Murcia), España

2 Departamento de Urología, Hospital General Universitario Reina Sofía, Murcia, España

${ }^{3}$ División de Medicina Preventiva y Salud Pública, Universidad de Medicina, Murcia, España

${ }^{4}$ Departamento de Medicina Preventiva, Hospital General

Universitario Reina Sofia, Murcia, España

${ }^{5}$ Centro de investigación operativa, Universidad Miguel Hernández, Elche (Alicante), España

6 Instituto Murciano de Investigación Biosanitaria (IMIB-Arrixaca), EI Palmar (Murcia), España

7 Departamento Anatomía Patológica Hospital General Universitario Reina Sofía, Murcia, España
}

Address for correspondence Carlos Sánchez Rodríguez, Avenida Marqués de los Vélez $72,3^{\circ} \mathrm{D}, \mathrm{CP} 30007$, Murcia, España (e-mail: charles_mh@hotmail.com).

Urol Colomb 2018;27:254-259.
Resumen
Objetivo La ratio digital del segundo y cuarto dedo de la mano (2D:4D) es un marcador de la exposición a andrógenos prenatal que presenta un dimorfismo sexual en mamíferos, siendo menor en hombres que en mujeres. El objetivo de nuestro estudio fue analizar la asociación entre la ratio 2D:4D (como marcador indirecto del ambiente hormonal intrauterino) y la severidad preoperatoria del cáncer de próstata (CaP).
Material y métodos Se trata de un estudio transversal que incluyó 118 pacientes intervenidos de CaP y con confirmación histológica por biopsia desde abril de 2007 hasta julio de 2015. A cada paciente se le realizó una exploración física y se midió desde
Palabras Clave
- neoplasia prostática
- ratio digital
- antígeno prostático específico
- biopsia
- cilindros
- severidad preoperatoria la base de la falange proximal hasta el extremo de la falange distal tanto en el cuarto como en el segundo dedo de la mano derecha. La asociación entre la ratio 2D:4D y los indicadores de severidad prequirúrgicos de $\mathrm{CaP}$ se realizaron mediante test de medias, correlación de Spearman y regresión lineal simple.
Resultados La ratio 2D:4D se asoció significativamente con el PSA prequirúrgico $(p=0,023)$ y con el porcentaje de cilindros afectados en la biopsia $(p=0,05)$
Conclusión Nuestros resultados sugieren que una mayor exposición androgénica prenatal, reflejado en una ratio 2D:4D disminuida, estaría asociada con una mayor severidad prequirúrgica del CaP.

received

June 26, 2017

accepted

September 18, 2017

published online

July 10,2018
DOI https://doi.org/

10.1055/s-0038-1651509.

ISSN 0120-789X.

eISSN 2027-0119.
Copyright (c) 2018, Sociedad Colombiana License terms de Urología. Publicado por Thieme Revinter Publicações Ltda., Rio de Janeiro, Brazil. Todos los derechos reservados.

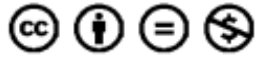




\author{
Abstract

\section{Keywords} \\ - neoplasm prostatic \\ - digit ratio \\ - prostate-specific \\ antigen \\ - biopsy \\ - cylinders \\ - preoperative severity
}

Objective Digit ratios, especially 2D:4D ratio, is a marker for prenatal androgen exposure that shows sexual dimorphism in mammals, being lower in men than in women. The aim of this study was to assess the associations between the $2 \mathrm{D}: 4 \mathrm{D}$ ratio (as an indirect marker for prenatal androgen environment) and preoperative severity of prostate cancer $(\mathrm{PCa})$.

Material and Methods This is a cross-sectional study of 118 operated PCa patients with confirmed biopsy of the tumor recruited between April 2007 and July 2015. All subjects underwent a physical examination, and the length from the bases of the second or fourth proximal phalanxes to the tips of the corresponding distal phalanxes on the right hand were measured. Associations between 2D:4D ratio and markers of PCa preoperative severity were evaluated using mean test scores, Spearman correlations and simple linear regression.

Results 2D:4D ratio was statistically significantly associated with preoperative PSA levels $(p=0.023)$ and the percentage of biopsy cylinders affected $(p=0.05)$.

Conclusions Our results suggest that higher prenatal androgen exposure, reflecting a lower 2D:4D ratio, may be associated with higher preoperative severity of PCa.

\section{Introducción}

El cociente entre la longitud del segundo y cuarto dedo (2D:4D) de la mano es un rasgo de dimorfismo sexual, presentando los hombres una ratio menor que las mujeres. ${ }^{1}$ Varios estudios de cohortes ${ }^{2,3}$ y un metaanálisis, ${ }^{4}$ han mostrado que la diferencia de género en la ratio de los dedos se asocia con la exposición de andrógenos prenatales. El cociente 2D:4D está inversamente relacionado a la exposición intrauterina de testosterona (T) y directamente relacionado a la de estradiol. ${ }^{2}$ Existe evidencia que afirma que la ratio $2 \mathrm{D}: 4 \mathrm{D}$ podría ser un marcador válido para los niveles hormonales del adulto (Ty estrógeno), ${ }^{3}$ aunque ese dato es controvertido. ${ }^{4}$ Por esa razón, el cociente 2D:4D se ha utilizado como un biomarcador no invasivo y retrospectivo para la exposición prenatal de andrógenos, y se ha correlacionado con una amplia gama de enfermedades como el autismo, ${ }^{5}$ así como la cognición visoespacial y la orientación sexual. ${ }^{6}$

Se conoce que el cáncer de próstata $(\mathrm{CaP})$ es una enfermedad hormonosensible. ${ }^{7}$ El desarrollo prenatal de la próstata depende de los andrógenos, específicamente de la dihidrotestosterona, y de la función de un receptor androgénico. ${ }^{8}$ Los niveles de testosterona sérica se asocian con la incidencia, ${ }^{9}$ la gravedad, ${ }^{10,11}$ y el pronóstico del CaP. ${ }^{11}$

También se ha estudiado la relación entre el cociente 2D:4D con la actividad del receptor androgénico y la repetición del triplete CAG en el gen de dicho receptor. Una disminución del triplete CAG está relacionado con un aumento del riesgo de $\mathrm{CaP}^{12,13}$ y de hiperplasia benigna de próstata. ${ }^{14,15}$

Sólo unos pocos estudios han investigado si la ratio 2D:4D se asocia con $\mathrm{CaP}^{16-18}$ y han sugerido que el cociente de dígitos podría ser un predictor de la presencia de esa enfermedad. Un estudio encontró que los pacientes con un cociente más bajo presentaban un riesgo aumentado de PSA elevado y por lo tanto un mayor riesgo de CaP en la biopsia. ${ }^{16}$ Otro estudio no descarta la existencia de una relación inversa entre el cociente y el riesgo de CaP. ${ }^{17}$ Por último, García- Cruz $\mathrm{y}$ colaboradores ${ }^{18}$ concluyen en su trabajo que un cociente 2D:4D bajo es un factor protector sobre el CaP, señalando que la elevada exposición intrauterina de testosterona podría estar relacionada con menor probabilidad de CaP en la vida adulta. En definitiva, observamos una discrepancia entre los resultados publicados obtenidos por los distintos autores.

Aunque la asociación entre el cociente 2D:4D y la presencia de CaP se ha estudiado, existen pocos datos sobre la relación entre el cociente 2D: 4D y la gravedad del CaP. ${ }^{19,20}$ Nuestra hipótesis es que una menor ratio 2D:4D, lo que sugiere una mayor exposición de andrógenos prenatales, se asocia con una mayor gravedad preoperatoria de la enfermedad.

\section{Material y métodos}

\section{Población del estudio}

Se trata de un estudio transversal con una muestra de todos los pacientes diagnosticados de CaP intervenidos quirúrgicamente en el Hospital General Universitario Reina Sofía de Murcia (HGURS) desde el abril de 2007 hasta julio de 2015. Para ello, una vez intervenidos mediante prostatectomía abierta 0 laparoscópica y una vez obtenida la confirmación histológica, se les contactó telefónicamente para explicarles el estudio e invitarles a participar en él. Si los pacientes aceptaban se concertaba una cita para realizar toda la recogida de variables. De un total de 313 pacientes telefoneados acudieron 124. De esos, entraron a formar parte del estudio 119 pacientes, ya que 5 no consiguieron terminar las encuestas.

Se citó a cada uno de los participantes en una consulta del hospital, donde se les realizó un examen físico, con medida de la longitud de los dedos incluida, y allí completaron cuestionarios sobre datos demográficos y hábitos de vida. Los participantes no obtuvieron compensación económica alguna y firmaron un consentimiento informado. El presente estudio tuvo la aprobación del Comité de Ética e Investigación Clínica del HGURS y de la Comisión de Ética de la Investigación de la Universidad de Murcia. 


\section{Examen Físico}

El índice de masa corporal (IMC) fue calculado para cada uno de los participantes. Se valoró la presencia de varicocele, hidrocele, quistes epididimarios y otras anomalías. Para la medición del tamaño de los dedos se utilizaron los de la mano derecha colocándolos planos en el borde de una mesa, con la palma posicionada en un ángulo de $100-120^{\circ}$ en relación con los dedos. Todas las medidas se tomaron por duplicado para aumentar la precisión usando un pie de rey digital (VWR International, LLC, Wext Chester, PA, USA). La medición abarcaba desde la base de la falange proximal hasta el extremo de la falange distal tanto en el cuarto como en el segundo dedo de la mano derecha.

\section{Cuestionario}

Cada paciente realizó un cuestionario con ayuda de un facultativo. Se recogió información sobre estilos de vida, incluyendo consumo de alcohol y tabaco, actividad física, etnia, estrés psicológico, toma de medicación, enfermedades o desórdenes generales y andrológicos previos. Con respecto al historial médico familiar, se obtuvo información sobre enfermedades (CaP), anomalías y desordenes reproductivos en los familiares de primer grado de todos los sujetos.

\section{Estudio microscópico y diagnóstico de CaP}

Los casos fueron sujetos intervenidos en nuestro Servicio de Urología por CaP y con confirmación histológica posterior. En nuestro centro, realizamos la biopsia de próstata transrectal ecodirigida con la técnica en sextante clásica más la obtención de tres cilindros de las zonas laterales de cada lóbulo, obteniendo un total de 12 cilindros. Cuando se trata de biopsias de saturación se indica una nueva estrategia con toma de 24 cilindros. Para el examen microscópico de la biopsia se procesó la muestra siguiendo los procedimientos habituales incluyéndola en parafina. A continuación, se seccionó a 3-4 micras para la tinción con hematoxilinaeosina. Para evaluar las lesiones se utilizó la escala de Gleason modificada. ${ }^{21}$ Posteriormente se agrupó a los pacientes según esa escala (Gleason $\leq 6,7 \mathrm{y}>7$ ).

\section{Análisis estadístico}

Se realizó un análisis descriptivo de las variables demográficas y clínicas de los participantes. Para las variables continuas se obtuvo la media, mediana, desviación típica (DT) y los percentiles 25 y 75 . Además, se analizó la normalidad de las variables mediante el test de Kolmogorov-Smirnov. Para las variables categóricas se obtuvo la frecuencia absoluta y el porcentaje.

Las asociaciones entre las características demográficas y clínicas con la ratio 2D:4D fueron analizadas mediante el test de la U de Mann-Whitney para variables categóricas dicotómicas, el test de Kruskal-Wallis para variables categóricas de más de 2 grupos, y la correlación de Spearman para variables continuas. Se realizó un análisis de regresión lineal simple entre las características clínicas continuas (PSA y cilindros afectados de la biopsia) y la ratio digital. Además, los datos atípicos a más de 3 DT fueron eliminados para el análisis de PSA y cilindros y la ratio digital.
Tabla 1 Características de los pacientes con cáncer de próstata $(n=118)$

\begin{tabular}{|l|l|l|l|l|l|}
\hline Características & M & DT & Me & P25 & P75 \\
\hline Edad (años) & 67,1 & 6,5 & 68,0 & 64,0 & 72,0 \\
\hline Peso (Kg) & 82,1 & 12,5 & 82,5 & 72,0 & 91,3 \\
\hline Altura (cm) & 166,7 & 6,3 & 166,5 & 162,0 & 171,0 \\
\hline IMC (Kg/m²) & 29,5 & 4,1 & 29,3 & 26,7 & 32,2 \\
\hline $\begin{array}{l}\text { Ratio 2D:4D } \\
\text { (mm) }\end{array}$ & 0,95 & 0,03 & 0,95 & 0,03 & 0,97 \\
\hline Etnia & & & $\mathbf{N}$ & $\%$ & \\
\hline \multicolumn{1}{|c|}{ Caucásico } & & & 111 & 98,2 & \\
\hline \multicolumn{1}{|c|}{ Otros } & & 2 & 1,8 & \\
\hline $\begin{array}{l}\text { Ha familiar } \\
\text { CaPa (Sí) }\end{array}$ & & 29 & 27,1 & \\
\hline $\begin{array}{l}\text { Consumo de } \\
\text { alcohol }^{\mathrm{b}} \text { (Sí) }\end{array}$ & & 106 & 92,2 & \\
\hline $\begin{array}{l}\text { Hábito } \\
\text { tabáquico }\end{array}$ & & 82 & 69,5 & \\
\hline
\end{tabular}

Abreviaciones: DT, desviación típica; IMC, índice de masa corporal; M, Media; Me, Mediana; P, percentil.

Todas las variables continuas tienen distribución normal.

A ¿Le han diagnosticado a algún familiar directo cáncer de próstata? B ¿Durante algún periodo de su vida ha consumido bebidas alcohólicas con una frecuencia de al menos 1 vez al mes?

C ¿Fuma o ha fumado a lo largo de su vida?

El índice de Gleason de la biopsia fue categorizado en 3 grupos $(<7,7 \mathrm{y}>7)$. Se obtuvieron el nomograma de Briganti (Briganti y col., 2012 ${ }^{22}$ ) y la clasificación de D'amico (D'amico y col., 1998 ${ }^{23}$ ) para evaluar el riesgo de afectación linfática (sí/no) y las categorías de riesgo del CaP (bajo/medio/alto) respectivamente.

Todos los test realizados fueron bilaterales, estableciendo el nivel de significación estadística en 0.05 . El estudio estadístico fue realizado mediante el programa estadístico IBM SPSS versión 21.0 (IBM Corporation, Armonk, NY).

\section{Resultados}

La - Tabla 1 muestra las principales características de nuestros pacientes. Eran varones con una edad media de 67,1 años (Desviación típica (DT); 6,5), un IMC medio de 29,5 (DT; 4,1) y de etnia caucásica en un $98,2 \%$. Un $69,5 \%$ eran fumadores y un $92,2 \%$ consumía alcohol al menos una vez al mes. La ratio 2D:4D media fue de $0,95 \mathrm{~mm}$ (DT; 0,03). Ninguna de esas variables se asocia de forma estadísticamente significativa con la ratio 2D:4D.

En la - Tabla 2, se muestran las variables de severidad preoperatorias y su relación con la ratio 2D:4D. Los valores medios de PSA preoperatorio fueron 7,5 ng/ml. Alrededor de tres cuartas partes de los participantes $(77,1 \%)$ tenían una puntuación de Gleason inferior a 7 en la biopsia de próstata. Un $38,8 \%$ de los cilindros estaban afectados de media en la biopsia. Después de la clasificación de D'Amico, el 55,1\% de los pacientes estaban en el grupo de bajo riesgo, el $28,8 \%$ 
Tabla 2 Asociación de las características demográficas y clínicas de los pacientes con cáncer de próstata y Ratio dígitos 2D:4D $(n=118)$

\begin{tabular}{|c|c|c|c|c|}
\hline Características & $\mathrm{Me}$ & P25 & P75 & $P^{\#}$ \\
\hline $\begin{array}{l}\text { PSA prequirúrgico } \\
\text { (ng/mL) }\end{array}$ & 7,3 & 6,0 & 11,3 & 0,023 \\
\hline \multirow{2}{*}{$\begin{array}{l}\text { Cilindros afectados } \\
\text { de biopsia (\%) }\end{array}$} & 38,8 & 16,7 & 50,0 & 0,050 \\
\hline & $\mathrm{N}$ & $\%$ & & \\
\hline Briganti & 39 & 33,1 & & 0,182 \\
\hline \multicolumn{5}{|l|}{ D'Amico } \\
\hline Bajo & 65 & 55,1 & & \\
\hline Medio & 34 & 28,8 & & \\
\hline Alto & 19 & 16,1 & & 0,482 \\
\hline \multicolumn{5}{|l|}{ Gleason biopsia } \\
\hline$<7$ & 91 & 77,1 & & \\
\hline 7 & 19 & 16,1 & & \\
\hline$>7$ & 8 & 6,8 & & 0,222 \\
\hline
\end{tabular}

Abreviaciones: Me, Mediana; P, percentil.

\#Correlación de Spearman para variables cuantitativas, U de MannWhitney y Kruskal-Wallis para variables cualitativas. Se han excluido los valores atípicos a más de 3 desviaciones estándar en las variables continuas.

Coeficientes de correlación de Spearman para PSA prequirúrgico 0,217 y cilindros afectados 0,181 .

tenían riesgo intermedio y el 16,1\% en el grupo de alto riesgo. Un tercio de los pacientes $(33,1 \%)$ tenían riesgo de invasión linfática según el nomograma de Briganti (aquellos con un riesgo calculado con el nomograma $>5 \%$ ).

Además la - Tabla 2, también refleja la asociación entre la ratio 2D:4D y las características clínicas preoperatorias de nuestros pacientes, observándose una asociación positiva significativa de los valores de la ratio 2D:4D y el PSA preoperatorio $(p=0,023)$ y con el porcentaje de cilindros afectados $(p=0,05)$.

También se ha realizado la regresión lineal simple con la ratio 2D:4D. El coeficiente de la ratio ha resultado significativo, por lo que se deduce la influencia de la ratio en el PSA preoperatorio ( $p=0,033$, coeficiente de regresión $0,203)$ y en el porcentaje de cilindros afectados $(p=0,035$, coeficiente de regresión 0,195).

\section{Discusión}

Nuestros hallazgos corroboran la hipótesis que sugiere la existencia de asociación entre los niveles prenatales de testosterona y la severidad del cáncer. Los resultados obtenidos respaldan que una menor ratio 2D:4D puede estar asociada con una mayor severidad prequirúrgica del CaP, en forma de niveles elevados de PSA y una mayor carga tumoral en la biopsia.

El cociente entre la longitud del segundo y cuarto dedo de la mano es un rasgo de dimorfismo sexual, presentando los hombres una ratio menor que las mujeres. ${ }^{1}$ Varios estudios han demostrado que la diferencia de género en la ratio de los dedos se asocia con la exposición de andrógenos prenatales. $^{2-4}$ En los seres humanos, el crecimiento y el patrón de los dedos de las manos y la diferenciación de las gónadas son controlados por los genes homeobox HOXA y HOXD. ${ }^{24}$ Por lo tanto, las hormonas gonadales fetales como la testosterona pueden influir en la morfología del dedo. ${ }^{25,26}$ Por ejemplo, una alta concentración de testosterona, lo que indica alta actividad testicular prenatal, conduce a una baja ratio digital. ${ }^{27}$ Recientemente, Lutchmaya y col., ${ }^{2}$ mostró que la ratio 2D:4D está negativamente asociado con los niveles de testosterona prenatal y está positivamente asociada con los niveles de estrógenos prenatales en humanos.

Es bien sabido que la testosterona y los receptores de andrógenos juegan un papel central en el crecimiento de la próstata y el desarrollo de CaP. ${ }^{7,8}$ La longitud de la repetición CAG del gen del receptor androgénico está asociado con la etiología del CaP. ${ }^{12,13}$ Manning y col., ${ }^{28}$ demostraron que la ratio 2D:4D de la mano derecha está positivamente correlacionado con el número de repeticiones CAG del receptor androgénico. También se ha descrito la relación entre la testosterona y la incidencia, ${ }^{9}$ la gravedad, ${ }^{10,11}$ y el pronóstico del CaP. ${ }^{11}$

Rahman y col., ${ }^{29}$ informó que la ratio digital es un marcador razonable para la evaluación del riesgo de CaP. En su estudio de casos y controles, una mayor ratio digital se relaciona con un efecto protector sobre el riesgo de CaP, en particular, en los pacientes con edad inferior a 60 años. Teniendo en cuenta esos estudios, es altamente sugerente que la ratio digital puede estar relacionado con el CaP.

En otro estudio sobre la relación entre la ratio digital y el CaP, Jung y col., ${ }^{16}$ propuso que la ratio del segundo y cuarto dígito de la mano derecha está relacionada con el PSA y con la presencia de CaP. Ellos mostraron una relación inversa significativa entre la ratio 2D:4D y el nivel de PSA y la presencia de CaP. Sin embargo, no encontraron que los resultados de la biopsia de próstata estuvieran correlacionados con la ratio digital porque el número de pacientes (366 pacientes) con CaP en su estudio, no era suficiente para revelar la relación de los dígitos con los hallazgos de la biopsia. Esos datos concuerdan con los resultados de nuestro estudio, en el que se aprecia una relación inversa estadísticamente significativa entre la ratio 2D:4D y los niveles de PSA preoperatorios. A su vez, hemos encontrado esa misma relación con la carga tumoral en forma de tanto por ciento de cilindros afectados en la biopsia prostática. Como Jung y col., ${ }^{16}$ no hemos encontrado relación entre la ratio 2D:4D y el Gleason de la biopsia, posiblemente por el bajo número de pacientes de la muestra.

Hasta la fecha, sólo cuatro estudios han investigado la relación entre la ratio digital y el riesgo de CaP. ${ }^{16-18,29} \mathrm{Sin}$ embargo, esos estudios no revelan la relación entre la ratio digital y la severidad preoperatoria del CaP, exceptuando lo expuesto anteriormente del trabajo de Jung y col., ${ }^{16}$ Respecto a la relación con la severidad postoperatoria, hemos encontrado dos trabajos en la literatura. ${ }^{19,20}$ En el primero de ellos, de Waters y col., ${ }^{19}$ no encuentran relación entre la ratio digital y la severidad del CaP pero no estudian ni el PSA 
ni el porcentaje de cilindros afectados. Además, concluyen que pueden existir factores de confusión que afecten al resultado así como un bajo tamaño muestral. En el segundo, Jin Kyu Oh y col., ${ }^{20}$ muestran asociación inversa entre la ratio 2D:4D y el volumen tumoral y grado de Gleason. Esos resultados respaldarían los obtenidos por nuestro grupo en cuanto a la asociación entre carga tumoral y ratio 2D:4D.

La ratio 2D:4D puede variar de acuerdo a lo que se mide, la mano seleccionada y el método utilizado (Peters y col. ${ }^{30}$ Manning y col; ${ }^{31}$ Voracek y col. ${ }^{27}$ Voracek y Loibl $^{32}$ ). Mediciones indirectas basadas en fotocopias o en el dibujo del contorno de los dedos puede distorsionar el cociente 2D:4D, siendo más bajo que el 2D:4D que se obtiene a partir de mediciones directas con los mismos puntos de referencia sobre los dedos, haciendo sospechar que pueda sesgar los resultados de varios estudios (Manning y col. ${ }^{31,33}$ ). En el presente estudio, se utilizó un método rápido de medición directa en base a referencias anatómicas (un método que recuerda a los métodos anteriores realizados por Peters y $\mathrm{col}^{30}{ }^{3}$ ), que proporciona mediciones cercanas a las que se pueden obtener de una radiografía de la mano.

Nuestra población era pequeña y limitada en edad y origen étnico y por lo tanto la generabilidad de los resultados es limitada. Otra limitación del estudio puede estar en relación con su diseño transversal que incluyó casos prevalentes, provocando que los casos más graves o con mayor mortalidad puedan no estar representados en el estudio, aunque conocemos que la morbimortalidad del $\mathrm{CaP}$ se da en un bajo porcentaje de pacientes. Otra limitación del estudio se relaciona con el proceso de reclutamiento de los sujetos del estudio. Se estableció contacto con los pacientes que habían sido tratados en el hospital entre los años 2007 y 2015 de CaP. Sólo alrededor de un tercio de ellos estaban disponibles para la medición y el estudio, no participando el resto de los pacientes en su mayoría por falta de tiempo. A pesar de que el nivel de participación voluntaria puede ser normal o incluso elevado si se compara con otros estudios clínicos, no podemos descartar un posible sesgo de selección, y por lo tanto se necesitan más estudios en diferentes poblaciones para confirmar esos hallazgos.

Hemos llegado a la conclusión de que los pacientes con menor ratio 2D:4D presentan una mayor severidad prequirúrgica del CaP en forma de niveles elevados de PSA y una mayor carga tumoral en la biopsia. Nuestros resultados, de ser confirmados por otros estudios, pueden proporcionar información adicional útil para contribuir a evaluar el riesgo de gravedad o severidad y pronóstico en dichos pacientes antes de recibir un tratamiento.

Conflictos de intereses

Los autores declaran que no existen conflictos de intereses.

Protección de personas y animales

Los autores declaran que para esta investigación, no se han realizado experimentos en seres humanos ni en animales.
Confidencialidad de los datos

Los autores declaran que han seguido los protocolos de su centro de trabajo sobre la publicación de datos de pacientes.

Derecho a la privacidad y consentimiento informado Los autores han obtenido el consentimiento informado de los pacientes y/o sujetos referidos en el artículo. Este documento obra en poder del autor de correspondencia.

\section{Bibliografía}

1 Phelps VR. Relative index finger length as a sex-influenced trait in man. Am J Hum Genet 1952;4(02):72-89

2 Lutchmaya S, Baron-Cohen S, Raggatt P, Knickmeyer R, Manning JT. 2nd to 4th digit ratios, fetal testosterone and estradiol. Early Hum Dev 2004;77(1-2):23-28

3 García-Cruz E, Huguet J, Piqueras M, Ribal MJ, Alcaraz A. Second to fourth digit ratio, adult testosterone level and testosterone deficiency. BJU Int 2012;109(02):266-271

4 Hönekopp J, Watson S. Meta-analysis of digit ratio 2D:4D shows greater sex difference in the right hand. Am J Hum Biol 2010;22 (05):619-630

5 Manning JT, Baron-Cohen S, Wheelwright S, Sanders G. The 2nd to 4th digit ratio and autism. Dev Med Child Neurol 2001;43(03):160-164

6 Collaer ML, Reimers S, Manning JT. Visuospatial performance on an internet line judgment task and potential hormonal markers: sex, sexual orientation, and 2D:4D. Arch Sex Behav 2007;36(02):177-192

7 Huggins C, Clark PJ. Quantitative studies of prostatic secretion: The effect of castration and of estrogen injection on the normal and on the hyperplastic prostate glands of dogs. J Exp Med 1940; 72(06):747-762

8 Heinlein CA, Chang C. Androgen receptor (AR) coregulators: an overview. Endocr Rev 2002;23(02):175-200

9 Boyle P, Koechlin A, Bota M, et al. Endogenous and exogenous testosterone and the risk of prostate cancer and increased prostate-specific antigen (PSA) level: a meta-analysis. BJU Int 2016;118(05):731-741

10 Pierorazio PM, Ferrucci L, Kettermann A, Longo DL, Metter EJ, Carter HB. Serum testosterone is associated with aggressive prostate cancer in older men: results from the Baltimore Longitudinal Study of Aging. BJU Int 2010;105(06):824-829

11 Porcaro AB, Petroziello A, Brunelli M, y col. High testosterone preoperative plasma levels independently predict biopsy Gleason score upgrading in men with prostate Cancer undergoing radical prostatectomy. Urol Int 2016;96(04):470-478

12 Giovannucci E. Is the androgen receptor CAG repeat length significant for prostate cancer? Cancer Epidemiol Biomarkers Prev 2002;11(10 Pt 1):985-986

13 Stanford JL, Just JJ, Gibbs M, y col. Polymorphic repeats in the androgen receptor gene: molecular markers of prostate cancer risk. Cancer Res 1997;57(06):1194-1198

14 Giovannucci E, Stampfer MJ, Chan A, y col. CAG repeat within the androgen receptor gene and incidence of surgery for benign prostatic hyperplasia in U.S. physicians. Prostate 1999;39(02):130-134

15 Roberts RO, Bergstralh EJ, Cunningham JM, y col. Androgen receptor gene polymorphisms and increased risk of urologic measures of benign prostatic hyperplasia. Am J Epidemiol 2004;159(03):269-276

16 Jung H, Kim KH, Yoon SJ, Kim TB. Second to fourth digit ratio: a predictor of prostate-specific antigen level and the presence of prostate cancer. BJU Int 2011;107(04):591-596

17 Muller DC, Giles GG, Manning JT, Hopper JL, English DR, Severi G. Second to fourth digit ratio (2D:4D) and prostate cancer risk in the 
Melbourne Collaborative Cohort Study. Br J Cancer 2011;105(03): 438-440

18 García-Cruz E, Piqueras M, Huguet J, y col. Higher second fourth digit ratio predicts higher incidence of prostate cancer in prostate biopsy. Arch Esp Urol 2012;65(09):816-821

19 Waters M, Rebholz CM, Wood B, Kuske A, McIntyre M, Sartor O. Second to fourth digit ratio and prostate cancer severity. Prostate Cancer Prostatic Dis 2013;16(01):107-110

20 Oh JK, Kim KH, Jung H, Yoon SJ, Kim TB. Second to fourth digit ratio: its relationship with core cancer volume and Gleason score in prostate biopsy. Int Braz J Urol 2012;38(05):611-619

21 Epstein JI, Allsbrook WC Jr, Amin MB, Egevad LL; ISUP Grading Committee. The 2005 International Society of Urological Pathology (ISUP) Consensus Conference on Gleason Grading of Prostatic Carcinoma. Am J Surg Pathol 2005;29(09):1228-1242

22 Briganti A, Larcher A, Abdollah F, y col. Updated nomogram predicting lymph node invasion in patients with prostate cancer undergoing extended pelvic lymph node dissection: the essential importance of percentage of positive cores. Eur Urol 2012;61(03):480-487

23 D'Amico AV, Whittington R, Malkowicz SB, y col. Biochemical outcome after radical prostatectomy, external beam radiation therapy, or interstitial radiation therapy for clinically localized prostate cancer. JAMA 1998;280(11):969-974

24 Voracek M, Manning JT. Length of fingers and penis are related through fetal Hox gene expression. Urology 2003;62(01):201

25 Manning JT, Scutt D, Wilson J, Lewis-Jones DI. The ratio of 2nd to 4th digit length: a predictor of sperm numbers and concentrations of testosterone, luteinizing hormone and oestrogen. Hum Reprod 1998;13(11):3000-3004

26 Williams TJ, Pepitone ME, Christensen SE, y col. Finger-length ratios and sexual orientation. Nature 2000;404(6777):455-456

27 Voracek M, Pietschnig J, Oeckher M. Finger, sex, and side differences in fingertip size and lack of association with imagebased digit ratio (2D:4D) measurements. Percept Mot Skills 2008; 107(02):507-512

28 Manning JT, Bundred PE, Newton DJ, Flanagan BF. The second to fourth digit ratio and variation in the androgen receptor gene. Evol Hum Behav 2003;24:399-405

29 Rahman AA, Lophatananon A, Stewart-Brown S, y col; UK Genetic Prostate Cancer Study Collaborators; British Association of Urological Surgeons' Section of Oncology. Hand pattern indicates prostate cancer risk. Br J Cancer 2011;104(01):175-177

30 Peters M, Mackenzie K, Bryden P. Finger length and distal finger extent patterns in humans. Am J Phys Anthropol 2002;117(03): 209-217

31 Manning JT, Fink B, Neave N, Caswell N. Photocopies yield lower digit ratios (2D:4D) than direct finger measurements. Arch Sex Behav 2005;34(03):329-333

32 Voracek M, Loibl LM. Scientometric analysis and bibliography of digit ratio (2D:4D) research, 1998-2008. Psychol Rep 2009;104 (03):922-956

33 Manning JT, Baron-Cohen S, Wheelwright S, Fink B. Is digit ratio (2D:4D) related to systemizing and empathizing? Evidence from direct finger measurements reported in the BBC internet survey. Pers Individ Dif 2010;48:767-771 